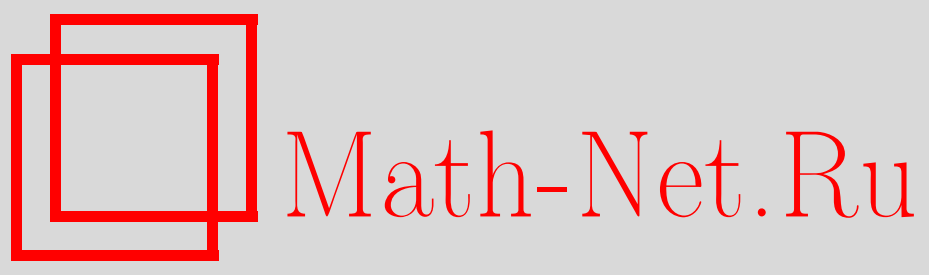

В. Н. Дубинин, К теореме о конечном приращении для комплексных полиномов, Матем. заметки, 2010, том 88, выпуск 5, 673-682

DOI: https://doi.org/10.4213/mzm8909

Использование Общероссийского математического портала Math-Net.Ru подразумевает, что вы прочитали и согласны с пользовательским соглашением http://www . mathnet.ru/rus/agreement

Параметры загрузки:

IP : 18.234 .156 .22

26 апреля 2023 г., $14: 47: 36$

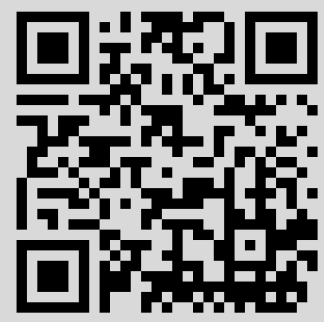


Том 88 выпуск 5 ноябрь 2010

УДК $512.62+517.54$

\section{K теореме о конечном приращении для комплексных полиномов}

\section{В. Н. Дубинин}

Для произвольного полинома $P$ степени не выше $n$ и любых точек $z_{1}$ и $z_{2}$ на комплексной плоскости устанавливаются оценки вида

$$
\left|P\left(z_{1}\right)-P\left(z_{2}\right)\right| \geqslant d_{n}\left|P^{\prime}\left(z_{1}\right)\right|\left|z_{1}-\zeta\right|,
$$

где $\zeta$ - один из корней уравнения $P(z)=P\left(z_{2}\right)$, а $d_{n}$ - положительная постоянная, зависящая только от числа $n$.

Библиография: 10 названий.

1. Введение и формулировки результатов. Комплексный полином $P$ степени $n$ определяется как алгебраический полином

$$
P(z)=c_{0}+c_{1} z+\cdots+c_{n} z^{n}
$$

с комплексными коэффициентами $c_{k}, k=0,1, \ldots, n, c_{n} \neq 0$. Общая теорема о конечном приращении дифференцируемых отображений в случае комплексных полиномов имеет вид

$$
\left|P\left(z_{1}\right)-P\left(z_{2}\right)\right| \leqslant\left|P^{\prime}(\xi)\right|\left|z_{1}-z_{2}\right|,
$$

где $z_{1}$ и $z_{2}$ - произвольные комплексные числа, а $\xi$ - некоторая точка отрезка $\left[z_{1}, z_{2}\right]$. Естественно поставить вопрос об уточнении и дополнении неравенства (1) с использованием специфики отображения, осуществляемого полиномом $P$. В частности, для каких точек $z_{1}$ и $z_{2}$ справедливо неравенство (1) с возможным положительным множителем в правой части, зависящим только от степени $n$, если точка $\xi$ является концом отрезка $\left[z_{1}, z_{2}\right]$ ? Имеет ли место неравенство, противоположное неравенству (1) и также с некоторым положительным множителем в правой части? Несмотря на обилие литературы по полиномам, указанный вопрос мало изучен (см., например, [1]-[3]). Отметим аналог неравенства (1) в случае, когда одна из точек $z_{k}$ является критической:

$$
|P(z)-P(\zeta)| \leqslant 4\left|P^{\prime}(z)\right||z-\zeta|
$$

Здесь $z$ - произвольная некритическая точка полинома $P$, а $\zeta$ - некоторая его критическая точка. Доказательство выписанного неравенства получено Смейлом [4],

Работа выполнена при финансовой поддержке Российского фонда фундаментальных исследований (грант № 08-01-00028) и ДВО РАН (грант № 09-I-П4-02).

(C) В. Н. Дувинин, 2010 
который предположил также, что константу 4 можно заменить на 1 либо $1-1 / n$ [3; § 7.2]. В недавней работе [5] поставлен вопрос о получении противоположного неравенства (с другой константой) и, в частности, установлено, что для некоторой критической точки $\zeta$ справедлива оценка

$$
|P(z)-P(\zeta)| \geqslant\left|\frac{P^{\prime}(z)}{n 4^{n}}\right||z-\zeta| .
$$

Следующее утверждение можно рассматривать как первый шаг в получении нижних оценок модуля приращения полинома для произвольных точек комплексной плоскости (не обязательно критических).

Теорема 1. Для любого полинома $P$ степени не выше $n$ и любых точек $z_{1} u z_{2}$ комплексной плоскости $\mathbb{C}$ существует точка $\zeta \in \mathbb{C}$ такал, что $P(\zeta)=P\left(z_{2}\right)$ u выполняется неравенство

$$
\left|P\left(z_{1}\right)-P\left(z_{2}\right)\right| \geqslant \frac{4^{1 / n-1}}{n}\left|P^{\prime}\left(z_{1}\right)\right|\left|z_{1}-\zeta\right| .
$$

В общем случае точку $\zeta$ в неравенстве (3) нельзя заменить на $z_{2}$. Например, для полинома

$$
P(z)=\frac{1-(1-z)^{n}}{n}=z+c_{2} z^{2}+\cdots,
$$

для точки $z_{1}=0$ и для точек $z_{2}$, достаточно близких к корням $P$, отличным от $z_{1}$, величина $\left|P\left(z_{2}\right) / z_{2}\right|$ принимает сколь угодно малые значения $\left(<4^{1 / n-1} / n\right)$. Доказательство теоремы 1 опирается на результаты Хеймана [6] и свойства диссимметризации областей [7]. Ранее диссимметризацию в оценках для полиномов применяли в работе [8]. Интересно отметить, что для полинома $P$ степени $n$ и произвольных точек $z_{1}, z_{2}$ легко устанавливается существование точки $\zeta$ такой, что $P(\zeta)=P\left(z_{1}\right)$ и выполняется неравенство

$$
\left|P\left(z_{1}\right)-P\left(z_{2}\right)\right| \geqslant\left|\frac{P^{\prime}(\zeta)}{n}\right|\left|\zeta-z_{2}\right| .
$$

Действительно, считая корни $\zeta_{k}, k=1, \ldots, n$, уравнения $P(\zeta)=P\left(z_{1}\right)$ различными, имеем для любых $z \in \mathbb{C}$

$$
1=\left|\sum_{k=1}^{n} \frac{P(z)-P\left(z_{1}\right)}{P^{\prime}\left(\zeta_{k}\right)\left(z-\zeta_{k}\right)}\right| \leqslant \sum_{k=1}^{n}\left|\frac{P(z)-P\left(z_{1}\right)}{P^{\prime}\left(\zeta_{k}\right)\left(z-\zeta_{k}\right)}\right|,
$$

что дает требуемое неравенство при некотором $\zeta=\zeta_{k}, 1 \leqslant k \leqslant n$. Равенство достигается в случае полинома $P(z)=1+z^{n}$ и точек $\zeta=z_{1}=1, z_{2}=0$.

Известная теорема покрытия Кебе-Бибербаха утверждает, что если функция $f(z)=z+c_{2} z^{2}+\cdots$ регулярна и однолистна в круге $|z|<1$, то образ круга $|z|<1$ при отображении этой функцией целиком покрывает круг $|w|<1 / 4$, т.е. круг один и тот же для всех функций такого рода. Соответствующая теорема для всего класса функций $f(z)=z+c_{2} z^{2}+\cdots$, регулярных в $|z|<1$, не верна, т.е. нельзя указать круга абсолютного радиуса и с центром в начале, который покрывался бы целиком образом круга $|z|<1$ при отображении каждой такой функцией. Тем не менее, из теоремы 1 непосредственно вытекает 
СлеДСТВИЕ 1. Для любого вещественного числа $R>0$ образ круга $|z|<R$ nри отображении полиномом $P(z)=z+c_{2} z^{2}+\cdots$ степени не выше $n$ иеликом покрывает круг $|w|<R 4^{1 / n-1} / n$.

Как показывает пример приведенного выше полинома $P$, указанное в следствии 1 накрытие не обязано быть $n$-кратным. Данное следствие по-существу эквивалентно полученной теореме о конечном приращении для комплексных полиномов. Интересно было бы найти радиус наибольшего круга в следствии 1, покрываемого кругом $|z|<R$ при отображении $P$.

Теорема 2. Пусть $P$ - полином степени не выше $n, z_{1} u z_{2}$ - произвольные точки комплексной плоскости $\mathbb{C}$, и пусть $\gamma$ - связная компонента прообраза отрезка $\left[P\left(z_{1}\right), P\left(z_{2}\right)\right]$ при отображении $P$, содержащая точку $z_{1}$. Тогда для любой точки $\zeta \in \gamma$ выполняется неравенство

$$
\left|P\left(z_{1}\right)-P\left(z_{2}\right)\right| \geqslant \frac{1}{n^{2}}\left|P^{\prime}\left(z_{1}\right)\right|\left|z_{1}-\zeta\right|
$$

Равенство в (4) достигается для полинома Чебишева $T_{n}(z)$, точек $z_{1}=1, \zeta=-1$ и для произвольной точки $z_{2}$, удовлетворяющей условию $T_{n}\left(z_{2}\right)=-1$.

Напомним, что в терминах конформных отображений полином

$$
T_{n}(z)=2^{n-1} z^{n}+\cdots
$$

определяется как суперпозиция обратной функции Жуковского, степенной функции и функции Жуковского:

$$
T_{n}(z)=\frac{1}{2}\left(\left(z+\sqrt{z^{2}-1}\right)^{n}+\left(z-\sqrt{z^{2}-1}\right)^{n}\right), \quad z \in \mathbb{C} .
$$

Отсюда, в частности, легко следует равенство в соотношении (4) для полинома $T_{n}(z)$ и точек, указанных в теореме 2. Доказательство теоремы 2 восходит в идейном плане к доказательству гипотезы Хеймана о покрытии вертикальных отрезков при конформном отображении круга [9]. В случае, когда континуум $\gamma$ в теореме 2 есть отрезок, неравенство (4) вытекает из классического неравенства Маркова. Немного сложнее увидеть, что неравенство Маркова содержится в теореме 2. Действительно, можно считать, что полином $P$ имеет вещественные коэффициенты, $P([-1,1])=[-1,1]$ и $P( \pm 1)= \pm 1$. Применяя теорему 2 к подходящему континууму из $P^{-1}([-1, P(x)])$ либо $P^{-1}([P(x), 1])$, получим неравенство Маркова

$$
\left|P^{\prime}(x)\right| \leqslant n^{2}
$$

где $\left|P^{\prime}(x)\right|=\max \left\{\left|P^{\prime}(z)\right|: z \in[-1,1]\right\},-1 \leqslant x \leqslant 1$. Можно показать, что из теоремы 2 следует усиление неравенства 2 с заменой константы $n 4^{n}$ на $n^{2}$. Сравнительно недавно Еременко [10] установил замечательное неравенство марковского типа для произвольного континуума, из которого вытекает, в частности, ослабленная версия неравенства (4) с дополнительным множителем $2^{1 / n}$ в его левой части, а также неравенство (3) теоремы 1 с меньшей константой в правой части. 
2. Доказательство теоремы 1. Можно считать, что степень полинома $P$ равна $n, z_{1} \neq z_{2}$ и $P^{\prime}\left(z_{1}\right) \neq 0$. Линейной заменой общий случай сводится к ситуации, когда $z_{2}=P\left(z_{2}\right)=0$ и, следовательно, неравенство (3) имеет вид

$$
\left|P\left(z_{1}\right)\right| \geqslant \frac{4^{1 / n-1}}{n}\left|P^{\prime}\left(z_{1}\right)\right|\left|z_{1}-\zeta\right| .
$$

Предположим, что неравенство $\left(3^{\prime}\right)$ не выполняется ни при каких $\zeta, P(\zeta)=0$, и пусть $\zeta_{1}, \ldots, \zeta_{m}$ - все различные корни полинома $P, m \leqslant n$. Введем следующие обозначения:

- пусть

$$
\alpha=\min _{1 \leqslant k \leqslant m}\left|z_{1}-\zeta_{k}\right|>\frac{n\left|P\left(z_{1}\right)\right|}{4^{1 / n-1}\left|P^{\prime}\left(z_{1}\right)\right|} ;
$$

- $D$ - плоскость $\mathbb{C}$ с разрезами вдоль лучей

$$
\left\{z: \arg \left(z-z_{1}\right)=\arg \left(\zeta_{k}-z_{1}\right),\left|z-z_{1}\right| \geqslant \alpha\right\}, \quad k=1, \ldots, m
$$

- $\varphi$ - функция, конформно и однолистно отображающая круг $|\omega|<1$ на область $D$ так, что $\varphi(0)=z_{1}, \varphi^{\prime}(0)>0$;

- $D^{*}$ - плоскость $\mathbb{C}$ с разрезами вдоль лучей

$$
\left\{z: \arg \left(z-z_{1}\right)=\frac{2 \pi(k-1)}{n},\left|z-z_{1}\right| \geqslant \alpha\right\}, \quad k=1, \ldots, n ;
$$

- $\varphi^{*}$ - функция, конформно и однолистно отображающая круг $|\omega|<1$ на $D^{*}$, $\varphi^{*}(0)=z_{1}, \varphi^{* \prime}(0)>0$.

По лемме 1.4 работы [7] существует диссимметризация области $D^{*}$ в подмножество области $D$. Применяя следствие 1.4 этой работы, получаем, что

$$
\left|\varphi^{\prime}(0)\right| \geqslant\left|\varphi^{* \prime}(0)\right|=\alpha 4^{1 / n}
$$

Обозначим через $F(\omega)$ одну из регулярных ветвей функции $\log P(\varphi(\omega))$ в круге $|\omega|<1$. Область $F(\{\omega:|\omega|<1\})$ пересекает любую вертикальную прямую по семейству интервалов суммарной длины $<2 \pi n$. Из теоремы Хеймана [6; теорема 4.10] вытекает неравенство

$$
\left|F^{\prime}(0)\right| \leqslant 4 n
$$

С другой стороны, с учетом (6) имеем неравенство

$$
\left|F^{\prime}(0)\right|=\left|\frac{P^{\prime}\left(z_{1}\right) \varphi^{\prime}(0)}{P\left(z_{1}\right)}\right| \geqslant\left|\frac{\alpha 4^{1 / n} P^{\prime}\left(z_{1}\right)}{P\left(z_{1}\right)}\right|,
$$

которое вместе с (5) и (7) приводит к противоречию. Теорема доказана.

Использование логарифма в доказательстве теоремы 1 можно избежать, применив к функции $P(\varphi(\omega))$ результат Хеймана о $p$-листных отображениях [6; теоремa 5.1]. 
3. Вспомогательные построения и утверждения. Следующие построения необходимы для доказательства теоремы 2. Пусть полином $P$ нормирован условиями $P(1)=1$ и $P^{\prime}(1) \neq 0$. Обозначим через $\mathscr{R}$ риманову поверхность функции $\mathscr{P}^{-1}$, обратной полиному $P$. Далее рассматриваем функцию $\mathscr{P}^{-1}$ как однозначную функцию, заданную на поверхности $\mathscr{R}$, и пусть $\mathscr{P}: \overline{\mathbb{C}}_{z} \rightarrow \mathscr{R}$ есть функция, обратная $\mathscr{P}-1$ в этом смысле. Под проекцией точки $W \in \mathscr{R}$ понимается точка $P(\mathscr{P}-1(W)) \in \overline{\mathbb{C}}_{w}$. Предположим, что луч $\{w: \operatorname{Im} w=0,1<\operatorname{Re} w<\infty\}$ не содержит критических значений полинома $P$ (т.е. точек $P(\zeta)$, для которых $P^{\prime}(\zeta)=0$ при некотором $\zeta$ ). Обозначим через $\mathscr{L}$ луч на поверхности $\mathscr{R}$, точнее жорданову кривую, однолистно лежащую над указанным выше лучом сферы $\overline{\mathbb{C}}_{w}$ и соединяющую точки $\mathscr{P}(1)$ и $\mathscr{P}(\infty)$. Пусть

$$
T=\left\{t_{k}\right\}_{k=0}^{m}, \quad 1=t_{0}<t_{1}<\cdots<t_{m-1}<t_{m}=\infty,
$$

- разбиение промежутка $1 \leqslant t \leqslant \infty$, содержащее все те значения $t$ из $1<t<\infty$, при которых эллипс $\gamma(t):=\{w:|w+1|+|w-1|=t+1 / t\}$ содержит, по крайней мере, одно критическое значение полинома $P$. Наконец, обозначим через $\mathscr{C}(t)$ замкнутую жордановую кривую на $\mathscr{R}$, пересекающую луч $\mathscr{L}$, лежащую над эллипсом $\gamma(t)$ и ориентированную соответственно с положительной ориентацией на проекции $\gamma(t)$, $1<t<\infty, t \notin T, c(t)$ - образ кривой $\mathscr{C}(t)$ при отображении $\mathscr{P}^{-1}$.

ЛЕмма 1. Приращение аргумента

$$
\Delta_{c(t)} \arg P(z)
$$

есть неубывающая функция от $t$ на множестве $\{t: 1<t<\infty, t \notin T\}$.

ДоказатеЛЬСтво. Пусть $1<t^{\prime}<t^{\prime \prime}<\infty, t^{\prime}, t^{\prime \prime} \notin T$. Точки $\mathscr{P}(1)$ и $\mathscr{P}(\infty)$ находятся по разные стороны от кривой $\mathscr{C}\left(t^{\prime}\right)$ и от кривой $\mathscr{C}\left(t^{\prime \prime}\right)$ на поверхности $\mathscr{R}$. Следовательно, непересекающиеся жордановые кривые $c\left(t^{\prime}\right)$ и $c\left(t^{\prime \prime}\right)$ отделяют точку 1 от $\infty$ на сфере $\overline{\mathbb{C}}_{z}$. Поэтому одна из кривых лежит во внутренности другой. Далее, двигаясь по лучу $\mathscr{L}$ от $\mathscr{P}(1)$ до $\mathscr{P}(\infty)$, сначала встречаем кривую $\mathscr{C}\left(t^{\prime}\right)$, а затем $\mathscr{C}\left(t^{\prime \prime}\right)$. Это означает, что кривая $c\left(t^{\prime}\right)$ лежит во внутренности кривой $c\left(t^{\prime \prime}\right)$. Следовательно, число $N_{t^{\prime}}$ нулей полинома $P$, лежащих внутри $c\left(t^{\prime}\right)$, не превосходит числа нулей $N_{t^{\prime \prime}}$ внутри $c\left(t^{\prime \prime}\right)$ (с учетом их кратностей). Осталось воспользоваться принципом аргумента:

$$
2 \pi N_{t}=\Delta_{c(t)} \arg P(z), \quad t=t^{\prime}, t^{\prime \prime}
$$

Лемма доказана.

Пусть $\gamma$ - связная компонента прообраза $P^{-1}([-1,1])$, содержащая точку $z=1$, и пусть точка $\zeta_{0} \in \gamma, \zeta_{0} \neq 1$. Введем обозначения

$$
W_{0}=\mathscr{P}\left(\zeta_{0}\right), \quad H=\left\{z: 1+\left(\zeta_{0}-1\right) \tau, 1 \leqslant \tau \leqslant \infty\right\} .
$$

Кривая $\mathscr{C}(t)$ разбивает односвязную риманову поверхность $\mathscr{R}$ на две области. Двигаясь в одной такой области от точки $\mathscr{C}(t) \cap \mathscr{L}$ вдоль жордановой дуги на $\mathscr{L}$ в направлении вещественной оси, мы встретим точку $\mathscr{P}(\infty)$. С другой стороны, мы встретим точку $W_{0}$, двигаясь в другой области от точки $\mathscr{C}(t) \cap \mathscr{L}$ сначала вдоль 
дуги на луче $\mathscr{L}$ до точки $\mathscr{P}(1)$, а затем вдоль кривой $\mathscr{P}(\gamma)$ до точки $W_{0}$. Итак, точки $W_{0}$ и $\mathscr{P}(\infty)$ расположены по разные стороны от кривой $\mathscr{C}(t)$ при любом $t$, $1<t<\infty, t \notin T$. Отсюда следует, что для каждого $k=1, \ldots, m$ двусвязная область

$$
\mathscr{G}_{k}=\bigcup_{t_{k-1}<t<t_{k}} \mathscr{C}(t)
$$

также отделяет точки $W_{0}$ и $\mathscr{P}(\infty)$. В то же время кривая $\mathscr{P}(H)$ соединяет эти точки. Поэтому для каждого $k=1, \ldots, m$ на кривой $\mathscr{P}(H)$ найдется, по крайней мере, одна жордановая дуга $\mathscr{H}_{k}$, лежащая в области $\mathscr{G}_{k}$ и соединяющая ее граничные компоненты. Таким образом, в принятых выше обозначениях справедлива следующая

Лемма 2. Для любого $k=1, \ldots, m$ область $\mathscr{G}_{k} \backslash \mathscr{H}_{k}$ односвязная.

Далее нам понадобится понятие емкости конденсатора (см., например, [7]). Для достаточно малых положительных $r$ и $\rho$ на сфере $\overline{\mathbb{C}}_{z}$ рассмотрим конденсаторы

$$
\begin{aligned}
& C(r)=(H,\{z:|z-1| \leqslant r\}), \\
& C(r, \rho)=\left(H \cup\left\{z:\left|z-\zeta_{0}\right| \leqslant \rho\right\} \cup\left\{z:|z| \geqslant \frac{1}{\rho}\right\}\right. \\
&\left.\cup \bigcup_{P^{\prime}(\widetilde{z})=0}\{z:|z-\widetilde{z}| \leqslant \rho\},\{z:|z-1| \leqslant r\}\right) .
\end{aligned}
$$

ЛЕмма 3. При фиксированном $r, 0<r<\left|\zeta_{0}-1\right|$, для емкостей конденсаторов справедливо равенство

$$
\lim _{\rho \rightarrow 0} \operatorname{cap} C(r, \rho)=\operatorname{cap} C(r) .
$$

ДокАЗАТЕЛЬСтво. Воспользуемся непрерывностью емкости и тем фактом, что добавление к пластинам конденсатора конечного числа точек не меняет емкости этого конденсатора:

$$
\lim _{\rho \rightarrow 0} \operatorname{cap} C(r, \rho)=\operatorname{cap}\left(H \cup \bigcup_{P^{\prime}(\widetilde{z})=0}\{\widetilde{z}\},\{z:|z-1| \leqslant r\}\right)=\operatorname{cap} C(r)
$$

(см. предложения 1.4 и 1.6 из статьи [7]). Лемма доказана.

Введем следующие ниже обозначения и дадим некоторые комментарии к ним:

- $\omega=\Psi(w)$ - функция в области $\overline{\mathbb{C}}_{w} \backslash[-1,1]$, обратная функции Жуковского $w=(\omega+1 / \omega) / 2$ и переводящая эллипсы $\gamma(t)$ в окружности $|\omega|=t, 1<t<\infty$;

- $\zeta=f_{k}(W)$ - однозначная ветвь функции $\zeta=\log \Psi(W)$, конформно и однолистно отображающая область $\mathscr{G}_{k} \backslash \mathscr{H}_{k}$ в "полосу"

$$
\Pi_{k}:=\left\{\zeta: \xi_{k-1}<\operatorname{Re} \zeta<\xi_{k}\right\}, \quad k=1, \ldots, m ;
$$

здесь $\xi_{k}=\log t_{k}, k=0,1, \ldots, m$; выбор такой ветви возможен ввиду леммы 2 ; при $k=m \Pi_{k}-$ полуплоскость;

- $u(z)$ - потенциальная функция конденсатора $C(r, \rho)$, т.е. вещественнозначная непрерывная на $\overline{\mathbb{C}}_{z}$ функция, равная нулю на первой пластине конденсатора $C(r, \rho)$, единице на второй и гармоническая в дополнении этих пластин; 
- $v_{k}(\zeta)$ - функция, определенная равенством

$$
v_{k}(\zeta)= \begin{cases}u\left(\mathscr{P}^{-1}\left(f_{k}^{-1}(\zeta)\right)\right), & \zeta \in f_{k}\left(\mathscr{G}_{k} \backslash \mathscr{H}_{k}\right), \\ 0, & \zeta \in \Pi_{k} \backslash f_{k}\left(\mathscr{G}_{k} \backslash \mathscr{H}_{k}\right),\end{cases}
$$

$k=1, \ldots, m$; доопределим функцию $v_{k}$ на $\partial \Pi_{k}$ по непрерывности; полученную при этом функцию будем вновь обозначать $v_{k}$; нетрудно видеть, что функция $v_{k}$ удовлетворяет условию Липшица в полосе $\bar{\Pi}_{k}, k=1, \ldots, m$, а функция $v_{1}$ к тому же равна единице на множестве

$$
f_{1}\left(\mathscr{P}(\{z:|z-1| \leqslant r\}) \cap \mathscr{G}_{1}\right) ;
$$

- $v_{k}^{*}(\zeta)$ - результат симметризации Штейнера функции $v_{k}(\zeta), \zeta \in \bar{\Pi}_{k}$, относительно вещественной оси (см. [6]); каждая функция $v_{k}^{*}(\zeta)$ липшицева в $\bar{\Pi}_{k}$ и равна нулю на множестве

$$
\left\{\zeta \in \bar{\Pi}_{k}:|\operatorname{Im} \zeta| \geqslant \pi n\right\}, \quad k=1, \ldots, m ;
$$

из леммы 1 вытекают неравенства

$$
v_{k}^{*}\left(\xi_{k}+i \eta\right) \leqslant v_{k+1}^{*}\left(\xi_{k}+i \eta\right), \quad-\infty<\eta<\infty, \quad k=1, \ldots, m-1 ;
$$

- $\zeta=F(z)$ - функция, конформно и однолистно отображающая единичный круг $|z|<1$ на полосу $|\operatorname{Im} \zeta|<\pi n$ так, что $F(0)=0, F^{\prime}(0)>0$;

- $\widetilde{r}$ - верхняя грань всех $r$, при которых множество

$$
F(\{z:|z|<r\}) \cap\{\zeta: \operatorname{Re} \zeta>0\}
$$

принадлежит результату симметризации Штейнера относительно вещественной оси множества

$$
f_{1}\left(\mathscr{P}(\{z:|z-1| \leqslant r\}) \cap \mathscr{G}_{1}\right) ;
$$

- $v(\zeta)$ - потенциальная функция конденсатора

$$
\widetilde{C}(\widetilde{r})=\left(\overline{\mathbb{C}}_{\zeta} \backslash\{\zeta:|\operatorname{Im} \zeta|<\pi n\}, F(\{z:|z| \leqslant \widetilde{r}\})\right)
$$

легко убедиться, что

$$
\begin{array}{lll}
\frac{\partial v}{\partial \xi}=0 & \text { на прямой } & \operatorname{Re} \zeta=0, \\
\frac{\partial v}{\partial \xi} \leqslant 0 & \text { на любой прямой } & \operatorname{Re} \zeta=\xi>0 ;
\end{array}
$$

определение конденсатора $\widetilde{C}(\widetilde{r})$ можно было бы упростить, взяв в качестве его второй пластины круг с центром в начале и подходящего радиуса; однако в этом случае существенно усложняется проверка условий (9); в нашем случае линии уровня потенциальной функции $v$ совпадают с линиями уровня функции $F$ (т.е. с кривыми $\left|F^{-1}(\zeta)\right|=$ const).

Для достаточно гладкой функции $\lambda$ на открытом множестве $\Omega \subset \mathbb{C}$ положим

$$
I(\lambda, \Omega)=\int_{\Omega}|\nabla \lambda|^{2} d \sigma .
$$


Лемма 4. Справедливо неравенство

$$
2 \sum_{k=1}^{m} I\left(v_{k}^{*}, \Pi_{k}\right) \geqslant I(v, \mathbb{C}) .
$$

ДокАЗАТЕЛЬСтво. Положим

$$
\begin{array}{cl}
G_{k}=\left\{\zeta \in \Pi_{k}:|\operatorname{Im} \zeta|<\pi n\right\}, & k=1, \ldots, m, \\
l_{k}=\left\{\zeta: \operatorname{Re} \zeta=\xi_{k},|\operatorname{Im} \zeta|<\pi n\right\}, & k=1, \ldots, m-1 .
\end{array}
$$

Для каждого $k, 1 \leqslant k \leqslant m$, имеем

$$
\begin{aligned}
I\left(v_{k}^{*}, \Pi_{k}\right) & =I\left(v_{k}^{*}, G_{k}\right)=I\left(v_{k}^{*}-v+v, G_{k}\right) \\
& =I\left(v_{k}^{*}-v, G_{k}\right)+I\left(v, G_{k}\right)+2 \iint_{G_{k}}\left[\frac{\partial\left(v_{k}^{*}-v\right)}{\partial \xi} \frac{\partial v}{\partial \xi}+\frac{\partial\left(v_{k}^{*}-v\right)}{\partial \eta} \frac{\partial v}{\partial \eta}\right] d \xi d \eta \\
& \geqslant I\left(v, G_{k}\right)-2 \int_{\partial G_{k}}\left(v_{k}^{*}-v\right) \frac{\partial v}{\partial n} d s,
\end{aligned}
$$

где $\partial / \partial n$ означает дифференцирование вдоль внутренней нормали к границе области $G_{k}$ (угловые точки исключаются). Учитывая соотношения (8) и $(9)$, получаем

$$
\begin{aligned}
\sum_{k=1}^{m} I\left(v_{k}^{*}, \Pi_{k}\right) & \geqslant \sum_{k=1}^{m} I\left(v, G_{k}\right)-2 \sum_{k=1}^{m} \int_{\partial G_{k}}\left(v_{k}^{*}-v\right) \frac{\partial v}{\partial n} d s \\
& =\sum_{k=1}^{m} I\left(v, G_{k}\right)-2 \sum_{k=1}^{m-1} \int_{l_{k}}\left[\left(v_{k}^{*}-v\right)\left(-\frac{\partial v}{\partial \xi}\right)+\left(v_{k+1}^{*}-v\right) \frac{\partial v}{\partial \xi}\right] d s \\
& =\sum_{k=1}^{m} I\left(v, G_{k}\right)+2 \sum_{k=1}^{m-1} \int_{l_{k}}\left(v_{k}^{*}-v_{k+1}^{*}\right) \frac{\partial v}{\partial \xi} d s \geqslant \sum_{k=1}^{m} I\left(v, G_{k}\right)=\frac{1}{2} I(v, \mathbb{C}) .
\end{aligned}
$$

Лемма доказана.

4. Доказательство теоремы 2. Достаточно рассмотреть случай $P^{\prime}\left(z_{1}\right) \neq 0$. Также можно считать, что $z_{1}=1, P\left(z_{1}\right)=1$ и $P\left(z_{2}\right)=-1$. Иначе рассмотрим полином

$$
Q(z)=2 \frac{P\left(z_{2}\right)-P\left(z+z_{1}-1\right)}{P\left(z_{2}\right)-P\left(z_{1}\right)}-1,
$$

для которого $Q(1)=1$ и $Q\left(\widetilde{z}_{2}\right)=-1, \widetilde{z}_{2}=z_{2}-z_{1}+1$. Если для этого полинома теорема верна, то найдется такая точка $\widetilde{\zeta}$, что $Q(\widetilde{\zeta})=-1$ и

$$
2 \geqslant\left|\frac{Q^{\prime}(1)}{n^{2}}\right||1-\widetilde{\zeta}|
$$

Это означает, что для полинома $P$ и точки $\zeta=\widetilde{\zeta}+z_{1}-1$ имеем $P(\zeta)=P\left(z_{2}\right)$ и выполняется неравенство (4).

В приведенных выше предположениях относительно полинома $P$ примем обозначения, введенные в п. 3. Следующая цепочка соотношений вытекает последовательно из конформной инвариантности интеграла Дирихле, теоремы Полиа и Сегё о симметризации функций (см. [6]) и леммы 4 :

$$
\operatorname{cap} C(r, \rho)=I(u, \mathbb{C}) \geqslant \sum_{k=1}^{m} I\left(v_{k}, \Pi_{k}\right) \geqslant \sum_{k=1}^{m} I\left(v_{k}^{*}, \Pi_{k}\right) \geqslant \frac{1}{2} I(v, \mathbb{C})=\frac{1}{2} \operatorname{cap} \widetilde{C}(\widetilde{r}) .
$$


Привлекая лемму 3, имеем в итоге

$$
\operatorname{cap} C(r) \geqslant \frac{1}{2} \operatorname{cap} \widetilde{C}(\widetilde{r}) .
$$

Для вычисления асимптотики емкости конденсаторов при $r \rightarrow 0$ воспользуемся известными формулами (см., например, [7; формулы $(1.6),(1.8)])$, в которых $r(B, a)$ означает внутренний радиус области $B$ относительно точки $a \in B$. В результате получаем

$$
\begin{aligned}
\operatorname{cap} C(r) & =-\frac{2 \pi}{\log r}-\frac{1}{2 \pi}\left(\log r\left(\mathbb{C}_{z} \backslash H, 1\right)\right)\left(\frac{2 \pi}{\log r}\right)^{2}+o\left(\left(\frac{1}{\log r}\right)^{2}\right) \\
& =-\frac{2 \pi}{\log r}-2 \pi\left(\log \left[4\left|\zeta_{0}-1\right|\right]\right)\left(\frac{1}{\log r}\right)^{2}+o\left(\left(\frac{1}{\log r}\right)^{2}\right), \quad r \rightarrow 0 .
\end{aligned}
$$

Далее, вторая пластина конденсатора $\widetilde{C}(\widetilde{r})$ представляет собой “почти круг" радиуса $\left(2\left|P^{\prime}(1)\right| r\right)^{1 / 2}(1+o(1))$ при $r \rightarrow 0$. Отсюда

$$
\begin{aligned}
\frac{1}{2} \operatorname{cap} \widetilde{C}(\widetilde{r})=- & \frac{\pi}{\log \left(2\left|P^{\prime}(1)\right| r\right)^{1 / 2}} \\
& -\pi(\log r(\{\zeta:|\operatorname{Im} \zeta|<\pi n\}, 0))\left(\frac{1}{\log \left(2\left|P^{\prime}(1)\right| r\right)^{1 / 2}}\right)^{2}+o\left(\left(\frac{1}{\log r}\right)^{2}\right) \\
= & -\frac{2 \pi}{\log \left(2\left|P^{\prime}(1)\right| r\right)}-4 \pi(\log (4 n))\left(\frac{1}{\log \left(2\left|P^{\prime}(1)\right| r\right)}\right)^{2}+o\left(\left(\frac{1}{\log r}\right)^{2}\right) \\
= & -\frac{2 \pi}{\log r}\left(1-\frac{\log \left(2\left|P^{\prime}(1)\right|\right)}{\log r}+o\left(\frac{1}{\log r}\right)\right)^{2} \\
& -4 \pi(\log (4 n))\left(\frac{1}{\log r}\right)^{2}+o\left(\left(\frac{1}{\log r}\right)^{2}\right) \\
= & -\frac{2 \pi}{\log r}-2 \pi\left(\log \left(\frac{8 n^{2}}{\left|P^{\prime}(1)\right|}\right)\right)\left(\frac{1}{\log r}\right)^{2}+o\left(\left(\frac{1}{\log r}\right)^{2}\right), \quad r \rightarrow 0 .
\end{aligned}
$$

Подставляя найденные асимптотические выражения в неравенство (10), приходим к заключению

$$
\left|\zeta_{0}-1\right| \leqslant \frac{2 n^{2}}{\left|P^{\prime}(1)\right|} .
$$

В наших предположениях полученное неравенство совпадает с неравенством (4) $\left(\zeta_{0}=\zeta\right)$. Случай равенства проверяется непосредственно. Теорема доказана.

\section{СПИСОК ЦИТИРОВАННОЙ ЛИТЕРАТУРЫ}

[1] G.V. Milovanović, D.S. Mitrinović, Th. M. Rassias, Topics in Polynomials: Extremal Problems, Inequalities, Zeros, World Sci. Publ., Singapore, 1994.

[2] P. Borwein, T. Erdélyi, Polynomials and Polynomial Inequalities, Grad. Texts in Math., 161, Springer-Verlag, New York, NY, 1995.

[3] Q. I. Rahman, G. Schmeisser, Analytic Theory of Polynomials, London Math. Soc. Monogr. (N.S.), 26, Oxford Univ. Press, Oxford, 2002.

[4] S. Smale, "The fundamental theorem of algebra and complexity theory", Bull. Amer. Math. Soc. (N.S.), 4:1 (1981), 1-36. 
[5] V. Dubinin, T. Sugawa, "Dual mean value problem for complex polynomials", Proc. Japan Acad. Ser. A Math. Sci., 85:9 (2009), 135-137.

[6] W. K. Hayman, Multivalent Functions, Cambridge Tracts in Math., 110, Cambridge Univ. Press, Cambridge, 1994.

[7] В.Н.Дубинин, "Симметризация в геометрической теории функций комплексного переменного", УМН, 49:1 (1994), 3-76.

[8] A.F. Beardon, D. Minda, T. W. Ng, "Smale's mean value conjecture and the hyperbolic metric", Math. Ann., 322:4 (2002), 623-632.

[9] В.Н. Дубинин, "О покрытии вертикальных отрезков при конформном отображении", Матем. заметки, 28:1 (1980), 25-32.

[10] A. Eremenko, "A Markov-type inequality for arbitrary plane continua", Proc. Amer. Math. Soc., 135:5 (2007), 1505-1510.

В. Н. Дубинин

Институт прикладной математики ДВО РАН

E-mail: dubinin@iam.dvo.ru
Поступило

27.01.2010 